\title{
SALUD PÚBLICA Y ADMINISTRACIÓN SANITARIA
}

\section{Decisión № 1786/CE DEL PARLAMENTO EUROPEO Y DEL CONSEJ 0 de 23 de septiembre de 2002 relativa a la adopción de un programa de acción comunitaria en el ámbito de la salud pública (2003-2008) Decision no. 1786/EC OF THE EUROPEAN PARLIAMENT AND OF THE COUNCIL of September $23^{\text {rd }}$, concerning the adoption of a program of community action in the field of public health (2003-2008)}

EL PARLAMENTO EUROPEO Y EL CONSEJO DE LA UNIÓN EUROPEA,

Visto el Tratado constitutivo de la Comunidad Europea y, en particular, su artículo 152,

Vista la propuesta de la Comisión (1),

Visto el dictamen del Comité Económico y Social (2),

Visto el dictamen del Comité de las Regiones (3),

De conformidad con el procedimiento establecido en el artículo 251 del Tratado (4), a la vista del texto conjunto aprobado por el Comité de Conciliación el 15 de mayo de 2002 ,

Considerando lo siguiente:

(1) La Comunidad ha adquirido el compromiso de promover y mejorar la salud, prevenir las enfermedades y atajar las amenazas potenciales para la salud con objeto de reducir la morbilidad y la mortalidad prematura evitable y la discapacidad acompañada de una merma de la actividad. Para contribuir al bienestar de los ciudadanos europeos, la Comunidad debe atender de manera coordinada y coherente las preocupaciones de su población acerca de los riesgos sanitarios y sus expectativas de un nivel elevado de protección de la salud. Por ello todas las actividades de la Comunidad relacionadas con la salud deben darse a conocer y ser transparentes, así como permitir la consulta y participación equilibradas de todos los agentes interesados, con el propósito de fomentar la mejora de los conocimientos y de los flujos de comunicación, posibilitando así una participación más amplia de las personas en las

(1) DO C 337 E de 28.11 .2000 , p. 122 y DO C 240 E de 28.8.2001, p. 168

(2) DO C 116 de 20.4 .2001$, p. 75.

(3) DO C 144 de 16.5 .2001$, p. 43

(4) Dictamen del Parlamento Europeo de 4 de abril de 2001 (DO C 21 de 24.1.2002, p. 161), Posición común del Consejo de 31 de julio de 2001 (DO C 307 de 31.10.2001, p. 27) y Decisión del Parlamen- decisiones que afectan a su salud. En este contexto, se debe atender al derecho de la población de la Comunidad a recibir información sencilla, clara y científicamente válida sobre las medidas para la protección de la salud y la prevención de enfermedades, con miras a mejorar la calidad de vida.

(2) La salud es prioritaria y debe garantizarse un alto nivel de protección para ella en la definición y aplicación de todas las políticas y actividades comunitarias. De acuerdo con el artículo 152 del Tratado, la Comunidad debe desempeñar un papel activo en este ámbito, adoptando medidas que no puedan adoptar los Estados miembros por sí solos, conforme al principio de subsidiariedad.

(3) Dentro del plan de salud pública presentado en la Comunicación de la Comisión, de 24 de noviembre de 1993, sobre el marco de actuación en el ámbito de la salud pública, se aprobaron ocho programas de acción, a saber:

- Decisión no 645/96/CE del Parlamento Europeo y del Consejo, de 29 de marzo de 1996, por la que se adopta un programa de acción comunitario de promoción, información, educación y formación en materia de salud en el marco de la acción en el ámbito de la salud pública (1996-2000) (5);

- Decisión no 646/96/CE del Parlamento Europeo y del Consejo, de 29 de marzo de 1996, por la que se adopta un plan de acción de lucha contra el cáncer en el marco de la acción en el

to Europeo de 12 de diciembre de 2001 (no publicada aún en el Diario Oficial). Decisión del Parlamento Europeo de 3 de julio de 2002 y Decisión del Consejo de 26 de junio de 2002

(5) DO L 95 de 16.4.1996, p. 1; Decisión cuya última modificación la constituye la Decisión no 521/2001/CE (DO L 79 de 17.3.2001, p. 1). 
ámbito de la salud pública (19962000) (6);

- Decisión no 647/96/CE del Parlamento Europeo y del Consejo, de 29 de marzo de 1996, por la que se adopta un programa de acción comunitaria relativo a la prevención del sida y de otras enfermedades transmisibles en el marco de la acción en el ámbito de la salud pública (1996-2000) (7);

- Decisión no 102/97/CE del Parlamento Europeo y del Consejo, de 16 de diciembre de 1996, por la que se adopta un programa de acción comunitaria relativo a la prevención de la toxicomanía en el marco de la acción en el ámbito de la salud pública (1996-2000) (8):

- Decisión no 1400/97/CE del Parlamento Europeo y del Consejo, de 30 de junio de 1997, por la que se adopta un Programa de acción comunitaria sobre vigilancia de la salud en el marco de la acción en el ámbito de la salud pública (1997-2001) (9);

- Decisión no 372/1999/CE del Parlamento Europeo y del Consejo, de 8 de febrero de 1999, por la que se aprueba un programa de acción comunitaria relativo a la prevención de lesiones en el marco de la acción en el ámbito de la salud pública (1999-2003) (10);

- Decisión no 1295/1999/CE del Parlamento Europeo y del Consejo, de 29 de abril de 1999, por la que se aprueba un programa de acción comunitaria sobre las enfermedades poco comunes en el marco de la acción en el ámbito de la salud pública (19992003) (11);

- Decisión no 1296/1999/CE del Parlamento Europeo y del Consejo, de 29 de abril de 1999, por la que se aprueba un programa de acción comunitaria sobre las enfermedades relaciona-

(6) DO L 95 de 16.4.1996, p. 9; Decisión cuya última modificación la constituye la Decisión no $521 / 2001 / C E$

(7) DO L 95 de 16.4 1996, p. 16; Decisión cuya última modificación la constituye la Decisión no 521/2001/CE

(8) DO L 19 de 22.1.1997, p. 25; Decisión modificada por la Decisión no 521/2001/CE.

(9) DO L 193 de 22.7.1997, p. 1; Decisión modificada por la Decisión no 521/2001/CE.

(10) DO L 46 de 20.2.1999, p. 1.

(11) DO L 155 de 22.6.1999, p. 1. das con la contaminación en el marco de la acción en el ámbito de la salud pública (1999-2001) (12).

Además, se aprobó la Decisión no 2119/98/CE del Parlamento Europeo y del Consejo, de 24 de septiembre de 1998, por la que se crea una red de vigilancia epidemiológica y de control de las enfermedades transmisibles en la Comunidad (13). En virtud de esta Decisión, la Comisión aprobó, el 22 de diciembre de 1999, la Decisión 2000/57/CE relativa al sistema de alerta precoz y respuesta para la vigilancia y control de las enfermedades transmisibles (14).

(4) Entre las restantes actividades incluidas en el marco de salud pública figura la Recomendación 98/463/CE del Consejo, de 29 de junio de 1998, sobre la idoneidad de los donantes de sangre y de plasma y el cribado de las donaciones de sangre en la Comunidad Europea (15), y la Recomendación 1999/519/CE del Consejo, de 12 de julio de 1999, relativa a la exposición del público en general a campos electromagnéticos (0 Hz a 300 GHz) (16).

(5) El plan de salud pública se revisó en la Comunicación de la Comisión, de 15 de abril de 1998, sobre el desarrollo de la política de salud pública en la Comunidad Europea, que indicó la necesidad de una nueva estrategia y un nuevo programa en el ámbito de la salud a la luz de las nuevas disposiciones del Tratado, los nuevos desafíos y la experiencia acumulada hasta la fecha.

(6) El Consejo, en sus Conclusiones, de 26 de noviembre de 1998, sobre el futuro marco de actuación de la Comunidad en materia de salud pública (17) y en su Resolución de 8 de junio de 1999 (18); el Comité Económico y Social, en su dictamen de 9 de septiembre de 1998 (19); el Comité de las Regiones, en su dictamen de 19 de noviembre de 1998 (20), así como el Parlamento Europeo, en su Resolución A4-0082/99 de 12 de marzo de 1999 (21), aplaudieron la Comunicación de la Comisión de 15 de

(12) DO L 155 de 22.6.1999, p. 7; Decisión modificada por la Decisión no 521/2001/CE.

(13) DO L 268 de 3.10.1998, p. 1.

(14) DO L 21 de 26.1.2000, p. 32.

(15) DO L 203 de 21.7.1998, p. 14.

(16) DO L 199 de 30.7.1999, p. 59.

(17) DO C 390 de 15.12.1998, p. 1.

(18) DO C 200 de 15.7.1999, p. 1.

(19) DO C 407 de 28.12.1998, p. 21

(20) DO C 51 de 22.2.1999, p. 53.

(21) DO C 175 de 21.6.1999, p. 135. 
abril de 1998 y defendieron la conveniencia de inscribir las acciones comunitarias en un programa global con una duración mínima de cinco años y tres objetivos generales, a saber: mejorar la información para el desarrollo de la salud pública, reaccionar rápidamente ante los riesgos sanitarios y abordar los factores determinantes de la salud mediante la promoción de la salud y la prevención de las enfermedades, con el apoyo de medidas intersectoriales y la utilización de todos los instrumentos apropiados previstos en el Tratado.

(7) El Consejo, en su Resolución, de 29 de junio de 2000, relativa al seguimiento de la Conferencia de Évora sobre determinantes de salud en la Unión Europea, consideró que las crecientes diferencias en la situación sanitaria y los efectos que ello supone para la salud dentro de cada Estado miembro, y entre ellos, requieren mayores esfuerzos coordinados a nivel nacional y comunitario; asimismo, acogió con agrado el compromiso de la Comisión de presentar una propuesta relativa a un nuevo programa de salud pública, que incluya líneas de acción específicas relativas a los determinantes de salud mediante la promoción de la salud y la prevención de las enfermedades, apoyada por una política intersectorial, y reconoció que para ello convendría desarrollar una base adecuada de conocimientos y establecer un sistema eficaz de seguimiento sanitario a tal efecto. Destacó la importancia de que la nueva estrategia comunitaria en materia de salud pública se funde en medidas relativas a determinantes de salud concretos ya tomadas en el marco de programas existentes, especialmente en lo que se refiere al tabaco, la alimentación y el alcohol y que es importante no sólo garantizar la continuidad de las actuaciones existentes sino también llevar adelante los trabajos al respecto de forma plenamente coherente y sistemática.

(8) El Consejo ratifica sus Conclusiones en materia de lucha contra el consumo de tabaco, de 18 de noviembre de 1999, en las que subrayaba la necesidad de desarrollar una estrategia global, e invitaba a la Comisión, entre otras cosas, a que reforzara la cooperación entre el sector sanitario y las políticas en otros ámbitos, con objeto de garantizar un nivel elevado de protección de la salud en estos ámbitos.

(9) El Consejo, de 18 de noviembre de 1999, aprobó por unanimidad una Resolución sobre la promoción de la salud mental.
(10) Según el informe de la OMS de 2000 sobre la salud en el mundo, las cinco principales causas de enfermedad (en años de vida con discapacidad) son: 1) los trastornos neuropsiquiátricos, 2) las enfermedades cardiovasculares, 3) los tumores malignos, 4) las lesiones involuntarias y 5) las enfermedades respiratorias. Las enfermedades infecciosas, como el sida, y la resistencia a los antibióticos se están convirtiendo en una amenaza para la salud de toda la población europea. Una importante función del programa sería determinar con más exactitud los principales riesgos de enfermedad de la Comunidad y, en particular, los principales determinantes sanitarios.

(11) El programa debe contribuir al intercambio de información sobre las normas de calidad reconocidas en el ámbito de la salud pública.

(12) Es indispensable recopilar, elaborar y analizar datos sobre la salud a escala comunitaria, con objeto de hacer posible un seguimiento eficaz del ámbito de la salud pública a escala comunitaria y de obtener una información objetiva, fiable, compatible y comparable, que se pueda intercambiar y que permita a la Comisión y a los Estados miembros mejorar la información al público y elaborar estrategias, políticas y acciones pertinentes con vistas a lograr un alto nivel de protección de la salud humana. Los datos del sector privado también deben tenerse en cuenta para completar el programa. Todas las estadísticas pertinentes deben desglosarse y analizarse por sexos.

(13) La Comunidad y los Estados miembros disponen de determinados medios y mecanismos en lo que respecta a la información y el seguimiento en el ámbito de la salud pública; en consecuencia, es indispensable garantizar un alto nivel de coordinación entre las acciones e iniciativas que toman la Comunidad y los Estados miembros para aplicar el programa, promover la cooperación entre los Estados miembros y acrecentar la eficacia de las redes existentes y futuras en el ámbito de la salud pública.

(14) Es fundamental que la Comisión garantice la eficacia y la coordinación de las medidas y acciones del programa, tanto a pequeña como a gran escala, así como el fomento de la cooperación entre los Estados miembros. Cualquier mecanismo estructural establecido con este objeto bajo los auspicios de la Comisión debe 
recopilar, vigilar y evaluar los datos, y desarrollar métodos de vigilancia de la salud y una base para reaccionar rápida y coordinadamente ante los riesgos sanitarios. Dichos mecanismos estructurales consistirían en un recurso central potenciado y contarían con la estrecha participación de las instituciones pertinentes designadas por los Estados miembros.

(15) En particular, es necesario garantizar, recurriendo a conocimientos especializados pertinentes, una adecuada coordinación duradera, en el ámbito de la información sanitaria, de las actividades destinadas a: determinar las necesidades de información, el desarrollo de indicadores, la recopilación de datos e información, las cuestiones de comparabilidad, el intercambio de datos e información con los Estados miembros y entre ellos, el desarrollo constante de bases de datos, análisis y una mayor divulgación de la información. Tal coordinación también debe garantizarse en el ámbito de la reacción rápida ante las amenazas a la salud, las actividades relacionadas con la vigilancia epidemiológica, el desarrollo de méto dos de vigilancia, el intercambio de información sobre directrices y sobre acciones, mecanismos y procedimientos de prevención y control.

(16) Es fundamental que la Comisión, mediante mecanismos estructurales apropiados, asegure la eficacia y la cohesión de las medidas y las acciones contenidas en el programa y promueva la cooperación entre los Estados miembros. En aras de un funcionamiento armonioso y eficaz de estos mecanismos estructurales, es necesario establecer una colaboración duradera con las autoridades sanitarias de los Estados miembros, observando, al mismo tiempo, las competencias de los Estados miembros.

(17) La Comisión debe formular, si procede, nuevas propuestas relativas al tipo de mecanismo estructural necesario para ejecutar la estrategia en el ámbito de la salud pública, en especial por lo que se refiere al seguimiento sanitario y a la reacción rápida ante los riesgos sanitarios.

(18) La finalidad global del programa de salud pública es contribuir a la consecución de un nivel elevado de salud y bienestar físico y mental y de una mayor igualdad en materia de salud en toda la Comunidad a través de medidas dirigidas a la mejora de la salud pública, evitar las enfermedades y trastornos humanos y eliminar las fuentes de peligro para la salud con el objetivo de luchar contra la morbilidad y la mortalidad prematura, teniendo en cuenta al mismo tiempo el sexo y la edad. Para alcanzar este objetivo, las acciones deben estar guiadas por la necesidad de aumentar la esperanza de vida sin discapacidad o enfermedad y promover la calidad de vida, así como reducir al máximo las consecuencias económicas y sociales de la enfermedad, reduciendo así las desigualdades sanitarias, teniendo en cuenta al mismo tiempo el enfoque regional frente a las cuestiones de salud. Debe otorgarse prioridad a las acciones de fomento de la salud dirigidas a las principales causas de enfermedad. El programa debe apoyar el desarrollo de una estrategia sanitaria integrada e intersectorial para asegurarse de que las políticas y acciones comunitarias contribuyen a la protección y a la promoción de la salud.

(19) Para lograr esto, el programa debe tener en cuenta la importancia de la educación, la formación y el establecimiento de redes.

(20) El Tratado dispone que en la definición y ejecución de todas las políticas y acciones comunitarias se garantice un alto nivel de protección de la salud humana. Todas las políticas comunitarias con incidencia en la salud deben vincularse estrechamente con la estrategia comunitaria en materia de salud pública. Tarea prioritaria en el marco del programa de salud pública será configurar criterios y metodologías para evaluar las propuestas de política y su aplicación. Al elaborar medidas en virtud del programa, y estrategias y acciones comunes con otros programas y acciones comunitarios pertinentes, debe velarse por que esas otras políticas y actuaciones comunitarias incorporen el aspecto de la salud y por que la política intersectorial las apoye.

(21) Alcanzar el objetivo global, así como los objetivos generales del programa, exige la cooperación efectiva de los Estados miembros, su pleno compromiso en la puesta en práctica de las acciones comunitarias y la participación de las instituciones, asociaciones, organizaciones y organismos en el ámbito sanitario, y de la población en general. Para garantizar la viabilidad y el uso eficaz de la inversión y capacidad comunitarias existentes, se deben utilizar las redes comunitarias y nacionales establecidas para compartir los conocimientos y la experiencia de los Estados miembros en relación con métodos eficaces de 
aplicación de las acciones preventivas y de promoción de la salud y con criterios de calidad. Debería asegurarse un diálogo con todos los interlocutores y agentes clave comprometidos en la mejora de la salud pública, e incorporar su experiencia a una base de conocimientos comunitaria eficaz y transparente en materia de salud. Se debe establecer una colaboración con los órganos y las organizaciones no gubernamentales que actúan en el ámbito de la salud a través de unos mecanismos adecuados, como los foros de salud.

(22) De conformidad con los principios de subsidiariedad y proporcionalidad establecidos en el artículo 5 del Tratado, en los ámbitos que no sean de su competencia exclusiva, como la salud pública, la Comunidad intervendrá sólo en la medida en que los objetivos de la acción puedan lograrse mejor, debido a la dimensión o a los efectos de la acción contemplada, a nivel comunitario. Los objetivos del programa no pueden ser alcanzados de manera suficiente por los Estados miembros debido a la complejidad, el carácter transnacional y la falta de control pleno, a nivel nacional, de los factores que inciden sobre la salud, por lo que el programa debe apoyar y complementar las acciones y medidas de los Estados miembros. El programa puede hacer importantes aportaciones al fomento de la salud y a los sistemas sanitarios en la Comunidad mediante el apoyo a estructuras y programas que refuercen las capacidades de las personas a nivel individual y las de las instituciones, asociaciones, organizaciones y organismos del ámbito de la salud, facilitando para ello el intercambio de las mejores prácticas y ofreciendo una base para el análisis común de los factores que afecten a la salud pública. El programa puede ser útil, además, en caso de amenazas para la salud pública de carácter transfronterizo por ejemplo en caso de enfermedades infecciosas, contaminación del medio ambiente o contaminación de alimentos en la medida en que requieren estrategias y acciones conjuntas. El programa permitirá a la Comunidad contribuir al cumplimiento de las obligaciones que le impone el Tratado en el ámbito de la salud pública, respetando plenamente las competencias de los Estados miembros respecto a la organización y prestación de servicios sanitarios y asistencia médica. La presente Decisión no excede de lo necesario para alcanzar dichos objetivos.
(23) Las medidas adoptadas en el marco del programa apoyan la estrategia sanitaria de la Comunidad y aportarán valor añadido comunitario al satisfacer las necesidades derivadas de las condiciones y estructuras establecidas por la acción comunitaria en otros ámbitos, abordar los últimos acontecimientos, las nuevas amenazas y los nuevos problemas respecto a los cuales la Comunidad estaría en mejores condiciones de actuar para proteger a su población, agrupar actividades emprendidas de forma un tanto aislada y con una incidencia limitada a nivel nacional y completarlas a fin de lograr resultados positivos para la población comunitaria, además de contribuir al refuerzo de la solidaridad y la cohesión en la Comunidad. La nueva estrategia sanitaria y el programa de acción en materia de salud pública deben ofrecer la oportunidad de seguir desarrollando la dimensión ciudadana de la política sanitaria de la Comunidad.

(24) Para que las acciones puedan abordar con eficacia y en cooperación con otras políticas y actividades comunitarias cuestiones y amenazas sanitarias de gran dimensión, evitando solapamientos, el programa debe prever la posibilidad de emprender acciones conjuntas con programas y acciones conexos de la Comunidad. Una utilización anticipativa de otras políticas comunitarias, como los Fondos Estructurales y la política social, podría influir de manera positiva en los factores determinantes de la salud.

(25) Para aplicar eficazmente las medidas y las acciones y lograr los efectos del programa buscados, es necesario que los datos recogidos sean comparables. La compatibilidad e interoperatividad de los sistemas y las redes de intercambio de información y datos para el desarrollo de la salud pública aportarían también una valiosa contribución y deben realizarse nuevos esfuerzos para alcanzar estos objetivos. El intercambio de información basada en datos comparables y compatibles reviste una importancia primordial.

(26) En general, las medidas y acciones del programa deben tener en cuenta el desarrollo de las nuevas tecnologías y las aplicaciones telemáticas; en particular, debe establecerse una estrecha coordinación con los proyectos definidos y realizados en el marco de la salud pública del programa integrado de acción relativo a una Europa electrónica (e-Europe) y con otros programas pertinentes, evitando los solapamien- 
tos, y tratando, en especial, de garantizar la igualdad en el acceso a la información sobre la salud.

(27) El Consejo Europeo de Feira de junio de 2000 aprobó el plan de acción «e-Europe 2002 - Una sociedad de la información para todos», que, en el marco de «Health Online», insta a los Estados miembros a desarrollar una infraestructura de sistemas de fácil manejo, validados e interoperativos sobre educación sanitaria, prevención de enfermedades y asistencia médica; es esencial que la nueva tecnología de la información se utilice para que los ciudadanos tengan el mayor acceso posible a la información sobre la salud.

(28) En la ejecución del programa se deben explotar al máximo los resultados generados por los programas de investigación comunitarios que prestan apoyo a la inves tigación en ámbitos cubiertos por el programa.

(29) Deben tenerse en cuenta las experiencias adquiridas a partir de las distintas cartas del ámbito de la salud. (30) En la puesta en práctica del programa comunitario, deben respetarse todas las disposiciones legales en el ámbito de la protección de los datos personales, así como establecerse mecanismos que garanticen la confidencialidad y seguridad de dichos datos.

(31) El programa debe tener una duración de seis años, con el fin de disponer de tiempo suficiente para aplicar las medidas necesarias a fin de lograr sus objetivos.

(32) Es esencial que la Comisión garantice la aplicación del programa en estrecha colaboración con los Estados miembros. A fin de obtener información y asesoramiento científicos para aplicar el programa, es deseable que se establezca una cooperación con científicos y expertos de prestigio internacional.

(33) Se debe asegurar la coherencia y la complementariedad entre las acciones previstas en el marco del programa y las previstas o desarrolladas en el contexto de otras políticas y acciones, sobre todo ante la necesidad de garantizar un alto nivel de protección de la salud humana en la definición y ejecución de todas las políticas y acciones comunitarias.

(34) Se espera que se establezca una cooperación estrecha y un mecanismo de consulta

(23) DO C 172 de 18.6.1999, p. 3.

(24) DO L 184 de 17.7.1999, p. 23. con los organismos comunitarios responsables de la evaluación de riesgos, el control y la investigación en los ámbitos de la alimentación y la seguridad alimentaria, la protección medioambiental y la seguridad de los productos.

(35) La presente Decisión establece, para toda la duración del programa, una dotación financiera que, con arreglo al punto $33 \mathrm{del}$ Acuerdo interinstitucional de 6 de mayo de 1999 entre el Parlamento Europeo, el Consejo y la Comisión sobre la disciplina presupuestaria y la mejora del procedimiento presupuestario (23), constituye la referencia privilegiada para la Autoridad Presupuestaria en el marco del procedimiento presupuestario anual. La dotación financiera debe ser acorde con las necesidades y los objetivos del programa.

(36) La financiación procedente de otras políticas comunitarias destinada a acciones conjuntas con arreglo al programa será adicional al marco financiero establecido para el programa.

(37) Es esencial que haya un margen de flexibilidad para poder reasignar los recursos y adaptar las actividades respetando la necesidad de transparencia así como los criterios de selección y clasificación de prioridades en función de la magnitud del riesgo o el efecto potencial, los resultados de la evaluación, las inquietudes de la población, la disponibilidad de las intervenciones o la posibilidad de desarrollarlas, la subsidiariedad, el valor añadido y la incidencia en otros sectores. No obstante, es necesario mantener un equilibrio entre los tres objetivos del programa, respetando una distribución equitativa de la dotación presupuestaria entre estos tres objetivos.

(38) Para alcanzar los objetivos del programa, es fundamental llevar a cabo acciones prácticas. Por consiguiente, al aplicar el programa y asignar los recursos disponibles, debe hacerse hincapié en la importancia de este tipo de acciones.

(39) Las medidas necesarias para la ejecución de la presente Decisión deben aprobarse con arreglo a la Decisión 1999/468/CE del Consejo, de 28 de junio de 1999, por la que se establecen los procedimientos para el ejercicio de las competencias de ejecución atribuidas a la Comisión (24).

ANALES Sis San Navarra 2003, Vol. 26, N 1, enero-abri 
(40) El Acuerdo sobre el Espacio Económico Europeo (Acuerdo EEE) prevé una mayor cooperación en el ámbito de la salud pública entre la Comunidad Europea y sus Estados miembros, por un lado, y los países de la Asociación Europea de Libre Comercio integrantes del Espacio Económico Europeo (países AELC/EEE), por otro. También se debe prever la apertura del programa a la participación de los países asociados de la Europa Central y Oriental con arreglo a las condiciones establecidas en los acuerdos europeos, en sus protocolos adicionales y en las decisiones de los consejos de asociación respectivos, de Chipre, financiada con créditos suplementarios conforme a los procedimientos que se acuerden con dicho país, así como de Malta y Turquía, financiada mediante créditos suplementarios con arreglo a las disposiciones del Tratado.

(41) Los países candidatos deben participar activamente en el desarrollo y la aplicación del nuevo programa y la sanidad de esos países, en particular sus problemas específicos, se debe atender desde una perspectiva estratégica.

(42) En el momento de la adhesión de nuevos Estados miembros, la Comisión debe informar sobre las consecuencias que dichas adhesiones tendrán para el programa.

(43) Se debe alentar la cooperación con los terceros países y las organizaciones internacionales competentes en el ámbito de la salud, como la OMS, el Consejo de Europa y la OCDE, no sólo en el ámbito de la recogida y análisis de datos (incluidos indicadores), sino también en el ámbito del fomento intersectorial de la salud con el fin de garantizar una buena relación costeeficacia, evitar solapamientos de actividades y programas y mejorar la sinergia y la interacción, teniendo en cuenta, sobre todo, las disposiciones de cooperación específicas, como las que existen entre la OMS y la Comisión.

(44) Las medidas adoptadas deben someterse a control y evaluación periódicos, incluidas evaluaciones externas independientes, a fin de incrementar el valor y la incidencia del programa. Debe ser posible adaptar o modificar el programa a la luz de estas evaluaciones y de los acontecimientos que se puedan producir en el contexto general de la acción de la Comunidad en el campo sanitario y los ámbitos relacionados con la salud. El Parlamento Europeo debe estar informado sobre los planes anuales de trabajo que redactará la Comisión.

(45) El programa de acción comunitario en el ámbito de la salud pública se basa en las actividades y los ocho programas desarrollados en el marco precedente, así como en los trabajos de la red de vigilancia epidemiológica y de control de las enfermedades transmisibles en la Comunidad, y persigue el conjunto de objetivos y medidas definidos en el marco de dichas acciones, en forma de una estrategia global e integrada de salud. Las decisiones relativas a estos ocho programas están cubiertas por el nuevo programa y, por consiguiente, deben derogarse con efectos a partir del 31 de diciembre de 2002.

DECIDE:

\section{Artículo 1}

\section{Establecimiento del programa}

1. La presente Decisión establece un programa de acción comunitario en el ámbito de la salud pública, en lo sucesivo denominado «el programa».

2. El programa se pondrá en práctica durante el período comprendido entre el 1 de enero de 2003 y el 31 de diciembre de 2008.

\section{Artículo 2}

\section{Propósito global y objetivos generales}

1. El programa, que complementará las políticas nacionales, tendrá por objeto proteger la salud humana y mejorar la salud pública.

2. Los objetivos generales del programa serán:

a) mejorar la información y los conocimientos a fin de fomentar la salud pública;

b) aumentar la capacidad de reaccionar rápida y coordinadamente ante los riesgos sanitarios;

c) fomentar la salud y prevenir las enfermedades actuando sobre los factores determinantes de la salud en todas las políticas y actividades

3. De este modo, el programa contribuirá a lo siguiente:

a) garantizar un nivel elevado de protección de la salud humana en la definición y ejecución de todas las políticas y acciones de la Comunidad, mediante el fomento de una estrategia de la salud integrada e intersectorial; 
b) reducir las desigualdades en materia de salud;

c) fomentar la cooperación entre los Estados miembros en los ámbitos regulados por el artículo 152 del Tratado.

\section{Artículo 3}

\section{Acciones y actividades de la Comunidad}

1. La consecución de los objetivos generales del programa, expuestos en el artículo 2, se llevará a cabo por medio de las acciones enumeradas en el anexo.

2. Estas acciones se ejecutarán en estrecha cooperación con los Estados miembros, mediante el apoyo a actividades de carácter transversal, que podrán utilizarse para ejecutar la totalidad o parte de las acciones, y combinarse cuando proceda. Estas actividades son las siguientes:

a) actividades relativas a los sistemas de observación y de reacción rápida:

i) actividades en red administradas mediante estructuras designadas por los Estados miembros y demás actividades de interés comunitario con fines de observación de la salud pública y suministro de información nacional, así como datos a escala comunitaria en apoyo de los objetivos del programa, ii) actividades para contrarrestar los riesgos sanitarios, incluidas las enfermedades graves, y reaccionar ante acontecimientos imprevistos, facilitar la investigación y coordinar las respuestas,

iii) preparación, creación y puesta en práctica de mecanismos estructurales adecuados que coordinen e integren las redes de observación sanitaria y de reacción rápida frente a riesgos sanitarios,

iv) el desarrollo de unos vínculos adecuados entre las acciones relativas a los sistemas de seguimiento y reacción rápida;

b) actividades sobre los factores determinantes de la salud: desarrollo y puesta en práctica de actividades de prevención de enfermedades y fomento de la salud integradas en todas las políticas comunitarias, en las que, cuando proceda, participen organizaciones no gubernamentales, proyectos y redes innovadores o experimentales entre instituciones y actividades nacionales;

c) actividades relativas a la legislación:

i) trabajos de preparación de instrumentos legislativos comunitarios en el ámbito de la salud pública, ii) evaluación de los efectos sanitarios de la legislación comunitaria,

iii) coordinación de la posición de la Comunidad y los Estados miembros en los foros en que se debaten temas relacionados con la salud;

d) actividades relativas a la consulta, el conocimiento y la información:

i) desarrollo y difusión a las autoridades competentes de los Estados miembros, a los profesionales de la sanidad y de otros sectores, así como, cuando proce$\mathrm{da}$, a otros interesados y al público general, de información y conocimientos sobre la salud, incluidos datos estadísticos, informes, estudios y análisis, así como asesoramiento sobre aspectos de interés común para la Comunidad y los Estados miembros,

ii) información y consultas sobre la salud y las cuestiones conexas a nivel comunitario, con la intervención de todos los participantes implicados, como las organizaciones de pacientes, los profesionales de la sanidad, el personal que presta atención sanitaria, los sindicatos, los interlocutores sociales y las ONG que actúan en el ámbito de la salud,

iii) intercambio de experiencia e información sobre los asuntos relativos a la salud entre la Comunidad y las autoridades y organismos competentes de los Estados miembros,

iv) fomento de la enseñanza y la formación profesional en el ámbito de la salud pública pertinentes para los objetivos del programa,

v) desarrollo y mantenimiento de redes para el intercambio de información sobre la mejor práctica en materia de salud pública y sobre la eficacia de las políticas sanitarias,

vi) obtención de información y asesoramiento de carácter científico de científicos y expertos de alto nivel,

vii) apoyo y fomento, por parte de la Comunidad y de los Estados miembros, de actividades relativas a buenas prácticas y orientaciones adecuadas en el ámbito de la salud pública basadas en datos científicos;

e) fomento de la coordinación a nivel europeo de organizaciones no gubernamentales que desarrollen actividades definidas como prioridades en el programa. Pueden estar operando tanto individualmente como en forma de varias asociaciones coordinadas. 
Artículo 4

\section{Estrategias y acciones conjuntas}

Para garantizar un nivel elevado de protección de la salud humana en la definición y ejecución de todas las políticas y acciones de la Comunidad, los objetivos del programa podrán desarrollarse como estrategias y acciones conjuntas mediante el establecimiento de vínculos con los programas y acciones comunitarios correspondientes, especialmente en los ámbitos de la protección de los consumidores, la protección social, la seguridad y la salud en el trabajo, el empleo, la investigación y el desarrollo tecnológico, el mercado interior, la sociedad y la tecno logía de la información, la estadística, la agricultura, la educación, el transporte, la industria y el medio ambiente, y con acciones emprendidas por el Centro Común de Investigación y los organismos comunitarios correspondientes, con quienes se impulsará la cooperación.

\section{Artículo 5}

\section{Aplicación del programa}

1. La Comisión garantizará la aplicación, en estrecha cooperación con los Estados miembros, de las acciones indicadas en el programa, de conformidad con lo dispuesto en el artículo 9 y garantizando que el programa se desarrolle de forma armoniosa y equilibrada.

2. Para prestar asistencia a la aplicación, la Comisión garantizará, mediante mecanismos estructurales pertinentes en los que participen estrechamente los Estados miembros, la coordinación y la integración de las redes de vigilancia de la salud y de reacción rápida ante riesgos sanitarios.

3. La Comisión y los Estados miembros adoptarán las medidas adecuadas, dentro de sus ámbitos de competencia respectivos, para garantizar que el programa se desarrolle eficientemente y para desarrollar los mecanismos establecidos a nivel comunitario y de los Estados miembros a fin de lograr los objetivos del programa. Cuidarán de que se proporcione información adecuada sobre las acciones que reciben un apoyo con arreglo al programa y de que la participación en las acciones cuya ejecución corresponda a las autoridades locales y regionales y las organizaciones no gubernamentales sea lo más amplia posible.

4. La Comisión, en estrecha cooperación con los Estados miembros, se impondrá como objetivo la comparabilidad de los datos y la información, $y$, en la medida de lo posible, la compatibilidad y la interoperatividad de los sistemas y redes de intercambio de información y datos en el ámbito de la salud.
5. En la puesta en práctica del programa, la Comisión, en colaboración con los Estados miembros, velará por el respeto de todas las disposiciones legales en el ámbito de la protección de los datos personales, así como por el establecimiento, cuando proceda, de mecanismos que garanticen la confidencialidad y seguridad de estos datos.

6. La Comisión, en estrecha cooperación con los Estados miembros, asegurará la transición entre las acciones que se desarrollen en el marco de los programas de salud pública adoptados por las Decisiones mencionadas en el artículo 13 , que contribuyen a las prioridades indicadas en ese programa, y las que se apliquen en el contexto del presente programa.

\section{Artículo 6}

\section{Coherencia y complementariedad}

La Comisión velará por la coherencia y la complementariedad de las acciones que se pongan en práctica en el marco del programa y de las que se desarrollen en el contexto de otras políticas y actividades de la Comunidad, incluidas las políticas mencionadas en el artículo 4. En particular, la Comisión determinará las propuestas que tienen una relación específica con los objetivos y las acciones del programa e informará al Comité mencionado en el artículo 9 sobre el modo en que se tengan en cuenta las consideraciones sanitarias en dichas propuestas, así como su incidencia prevista sobre la salud.

\section{Artículo 7}

\section{Financiación}

1. La dotación financiera para la ejecución del programa durante el período contemplado en el artículo 1 queda fijada en 312 millones de euros.

Los gastos de asesoramiento técnico y administrativo relacionado con los mecanismos estructurales mencionados en el inciso iii) de la letra a) del apartado 2 del artículo 3 y las actividades derivadas correrán a cargo del presupuesto global del programa.

2. La Autoridad Presupuestaria autorizará los créditos anuales dentro de los límites de las perspectivas financieras.

\section{Artículo 8}

\section{Medidas de aplicación}

1. Las medidas necesarias para la ejecución de la presente Decisión, relativas a las materias que se enumeran a continuación, se aprobarán con arreglo al procedimiento de gestión contemplado en el apartado 2 del artículo 9: 
a) el plan anual de trabajo para la aplicación del programa, que fijará las prioridades y las acciones que deberán llevarse a cabo, incluida la asignación de los recursos;

b) las medidas, criterios y procedimientos para seleccionar y financiar dichas acciones;

c) las medidas para aplicar las estrategias y acciones conjuntas a que se refiere el artículo 4;

d) las medidas de evaluación del programa a que se refiere el artículo 12;

e) las medidas relativas a la preparación de cualquier mecanismo estructural para la coordinación de la vigilancia de la salud y la reacción rápida ante riesgos sanitarios;

f) los métodos de transmisión, intercambio y comunicación de información y de reacción rápida ante riesgos sanitarios con arreglo al programa, sin perjuicio de las medidas de aplicación que se practiquen con arreglo a la no Decisión 2119/98/CE.

2. Las medidas necesarias para la ejecución de la presente Decisión relativas a todas las demás materias se aprobarán con arreglo al procedimiento consultivo contemplado en el apartado 3 del artículo 9 .

\section{Artículo 9}

\section{Comité}

1. La Comisión estará asistida por un Comité.

2. En los casos en que se haga referencia a este apartado, serán de aplicación los artículos 4 y 7 de la Decisión 1999/468/CE, observando lo dispuesto en su artículo 8. El plazo contemplado en el apartado 3 del artículo 4 de la Decisión 1999/468/CE quedará fijado en dos meses.

3. En los casos en que se haga referencia a este apartado, serán de aplicación los artículos 3 y 7 de la Decisión 1999/468/CE, observando lo dispuesto en su artículo 8.

4. El Comité aprobará su reglamento interno.

\section{Artículo 10}

Participación de los países AELC/EEE, los países asociados de la Europa Central y Oriental, Chipre, Malta y Turquía

Este programa estará abierto a la participación de:

a) Ios países AELC/EEE, de conformidad con las condiciones establecidas en el Acuerdo EEE;

b) Ios países asociados de la Europa Central y Oriental, de conformidad con las condicio- nes establecidas en los acuerdos europeos en sus protocolos adicionales y en las decisiones de los respectivos consejos de asociación;

c) Chipre, financiada mediante créditos suplementarios de acuerdo con los procedimientos que se determinen con ese país;

d) Malta y Turquía, financiada mediante créditos suplementarios de acuerdo con las disposiciones del Tratado.

\section{Artículo 11}

\section{Cooperación internacional}

Durante la puesta en práctica del programa se fomentará, de conformidad con el procedimiento que establece el apartado 3 del artículo 9, la cooperación con terceros países y con las organizaciones internacionales competentes en el ámbito de la salud pública, en particular la Organización Mundial de la Salud, el Consejo de Europa y la Organización de Cooperación y Desarrollo Económicos, o las que puedan tener una incidencia en la salud pública, tales como la Organización Mundial del Comercio y la Organización de las Naciones Unidas para la Agricultura y la Alimentación. En especial, el sistema de información sanitaria y la capacidad de responder a riesgos sanitarios deberán coordinarse, siempre que sea oportuno y posible, con las actividades de la Organización Mundial de la Salud.

\section{Artículo 12}

\section{Control, evaluación y difusión de resultados}

1. La Comisión, en estrecha colaboración con los Estados miembros, someterá a seguimiento regular, en su caso asistida por expertos, la aplicación de las acciones del programa a la vista de los objetivos. Informará de ello todos los años al Comité. La Comisión transmitirá copia de sus principales conclusiones al Parlamento Europeo y al Consejo.

2. A petición de la Comisión, los Estados miembros facilitarán información sobre la aplicación y la incidencia de este programa.

3. A más tardar a finales del cuarto año del programa, la Comisión encargará a expertos independientes cualificados la evaluación de la ejecución y de los logros de los tres primeros años del programa. Asimismo evaluará los efectos conseguidos sobre la salud y la eficacia del uso de los recursos, así como la coherencia y la complementariedad con otros programas, acciones e iniciativas pertinentes desarrollados en virtud de otras políticas y actividades comunitarias. La Comisión comunicará las conclusiones de dicha evaluación, acompañadas de sus 
observaciones, al Parlamento Europeo, al Consejo, al Comité Económico y Social y al Comité de las Regiones. La Comisión presentará asimis mo a estas instituciones y organismos, a más tardar a finales del año siguiente al de la finalización del programa, un informe definitivo sobre la ejecución del mismo.

4. La Comisión hará públicos tanto los resultados de las acciones acometidas como los informes de evaluación.

\section{Artículo 13}

\section{Derogación}

Quedan derogadas las Decisiones siguientes con efectos a partir del 31 de diciembre de 2002: Decisión no 645/96/CE, Decisión no 646/96/CE, Decisión no 647/96/CE, Decisión no 102/97/CE, Decisión no 1400/97/CE, Decisión no
372/1999/CE, Decisión no 1295/1999/CE, Decisión no 1296/1999/CE.

\section{Artículo 14}

\section{Entrada en vigor}

La presente Decisión entrará en vigor el día de su publicación en el Diario Oficial de las Comunidades Europeas.

Hecho en Bruselas, el 23 de septiembre de 2002.

Por el Parlamento Europeo

El Presidente

P. COX

Por el Consejo

El Presidente

M. FISCHER BOEL 
